\title{
Validation and ranking of seven staging systems of hepatocellular carcinoma
}

\author{
ZHAN-HONG CHEN ${ }^{1,2^{*}}$, YING-FEN HONG $^{1 *}$, JINXIANG LIN $^{1 *}$, XING LI $^{1}$, DONG-HAO WU ${ }^{1}$, \\ JING-YUN WEN ${ }^{1}$, JIE CHEN $^{1}$, DAN-YUN RUAN ${ }^{1}$, QU LIN ${ }^{1}$, MIN DONG $^{1}$, LI WEI $^{1}$, \\ TIAN-TIAN WANG ${ }^{1}$, ZE-XIAO LIN $^{1}$, XIAO-KUN MA ${ }^{1}$, XIANG-YUAN WU ${ }^{1}$ and RUIHUA XU ${ }^{2}$ \\ ${ }^{1}$ Department of Medical Oncology and Guangdong Key Laboratory of Liver Disease, \\ The Third Affiliated Hospital of Sun Yat-sen University, Guangzhou, Guangdong 510630; \\ ${ }^{2}$ Department of Medical Oncology of Sun Yat-sen University Cancer Center, State Key Laboratory of Oncology in \\ South China, Collaborative Innovation Center for Cancer Medicine, Guangzhou, Guangdong 510060, P.R. China
}

Received October 8, 2015; Accepted March 17, 2017

DOI: $10.3892 / \mathrm{ol} .2017 .6222$

\begin{abstract}
The aim of the present study was to evaluate the ability of seven staging systems to predict 3-and 6-month and cumulative survival rates of patients with advanced hepatitis $\mathrm{B}$ virus (HBV)-associated hepatocellular carcinoma (HCC). Data were collected from 220 patients with HBV-associated HCC who did not receive any standard anticancer treatment. Participants were patients at The Third Affiliated Hospital of Sun Yat-sen University from September 2008 to June 2010. The participants were classified according to the Chinese University Prognostic Index (CUPI), the Cancer of the Liver Italian Program (CLIP), Japan Integrated Staging (JIS), China Integrated Score (CIS) systems, Barcelona Clinic Liver Cancer (BCLC), Okuda and tumor-node-metastasis (TNM) staging systems at the time of diagnosis and during patient follow-up. The sensitivity and specificity of the predictive value of each staging system for 3- and 6-month mortality were analyzed by relative operating characteristic (ROC) curve analysis with a non-parametric test being used to compare the area under curve (AUC) of the ROC curves. In addition, log-rank tests
\end{abstract}

Correspondence to: Professor Xiang-Yuan Wu, Department of Medical Oncology and Guangdong Key Laboratory of Liver Disease, The Third Affiliated Hospital of Sun Yat-sen University, 600 Tianhe Road, Tianhe, Guangzhou, Guangdong 510630, P.R. China

E-mail: wuxiangy@mail.sysu.edu.cn

Professor Ruihua Xu, Department of Medical Oncology of Sun Yat-sen University Cancer Center, State Key Laboratory of Oncology in South China, Collaborative Innovation Center for Cancer Medicine, 651 Dongfengdong Road, Yuexiu, Guangzhou, Guangdong 510060, P.R. China

E-mail: xurh@sysucc.org.cn

*Contributed equally

Key words: hepatocellular carcinoma, hepatitis B virus, neoplasm staging, survival, prognosis and Kaplan-Meier estimator survival curves were applied to compare the overall survival rates of the patients with HCC defined as advanced using the various staging systems, and the Akaike information criterion (AIC) and likelihood ratio tests (LRTs) were used to evaluate the predictive value for overall survival in patients with advanced HCC. Using univariate and multivariate Cox's model analyses, the factors predictive of survival were also identified. A total of 220 patients with $\mathrm{HBV}$-associated HCC were analyzed. Independent prognostic factors identified by multivariate analyses included tumor size, $\alpha$-fetoprotein levels, blood urea nitrogen levels, the presence or absence of portal vein thrombus, Child-Pugh score and neutrophil count. When predicting 3-month survival, the AUCs of CLIP, CIS, CUPI, Okuda, TNM, JIS and BCLC were 0.806, $0.772,0.751,0.731,0.643,0.754$ and 0.622 , respectively. When predicting 6-month survival, the AUCs of CLIP, CIS, CUPI, Okuda, TNM, JIS and BCLC were 0.828, 0.729, 0.717, 0.692, $0.664,0.746$ and 0.575 , respectively. For 3 -month mortality, the prognostic value of CLIP ranked highest, followed by CIS; for 6-month mortality, the prognostic value of CLIP also ranked highest, followed by JIS. No significant difference between the AUCs of CLIP and CIS ( $\mathrm{P}>0.05)$ in their predictive value for 3-month mortality was observed. The AUC of CLIP was significantly higher compared with that of the other staging systems $(\mathrm{P}<0.05)$ for predicting 6-month mortality. The $\chi^{2}$ values from the LRTs of CLIP, CIS, CUPI, Okuda, TNM, JIS and BCLC were 75.6, 48.4, 46.7, 36.0, 21.0, 46.8 and 7.24, respectively. The AIC values of CLIP, CIS, CUPI, Okuda, TNM, JIS and BCLC were 1601.5, 1632.3, 1629.9, 1641.1, 1654.8, 1627.4 and 1671.1, respectively. CLIP exhibited the highest $\chi^{2}$ value and lowest AIC value, indicating that CLIP has the highest predictive value of cumulative survival rate. In the selected patients of the present study, CLIP was the staging system best able to predict 3- and 6-month and overall survival rates. CIS ranked second in predicting 3-month mortality.

\section{Introduction}

Hepatocellular carcinoma (HCC) is one of the most common malignancies in the world, with high incidence and mortality 
rates (1). Statistically among patients with HCC, males account for a higher proportion than females, and $\mathrm{HCC}$ is now the second leading cause of cancer-associated mortality worldwide in men (1). Globally, 50\% of patients with HCC are from China (1), where HCC occupies the third reason for cancer-associated mortality (2). In China, the leading cause of HCC (>80\%) is infection with hepatitis B virus (HBV) (3). As the patients with HCC are often diagnosed at an advanced stage, the majority of them are not able to receive curative therapies, including ablation, transplant and surgery. Although novel antitumor agents and clinical trials frequently emerge, the overall survival of the patients with HCC at advanced stage remains poor, and the life expectancy is too short to assess the effectiveness of novel anticancer agents (4-7). Life expectancy $>3$ months is an inclusion criterion for the majority of systemic drug trials in advanced HCC $(4,5)$. Eligibility for clinical trials is usually determined by Child-Pugh score and performance status (4,5). Alternative staging systems may be better at predicting survival and selecting patients for clinical trials in advanced HCC $(7,8)$.

Clinical staging aims to predict survival outcomes and assist in deciding the optimal therapeutic options for patients with cancer. However, HCC differs from other types of cancer in that its prognosis and treatment depend on two aspects: The tumor burden and liver function (6-9). Previous studies have developed staging systems that include these two features (6-11). Over previous decades, a number of staging systems have been developed based on specific populations of local regions (10-14). Numerous studies have attempted to identify a universally accepted staging system and prognosis assessment of HCC remains controversial $(6-11,15)$. It appears that different staging systems possess particular applicability among different groups of patients with HCC (10-14). Existing data provide a small amount of information (15) regarding which staging system is the most suitable candidate to accurately predict the prognosis of the patients with advanced HCC, which would assist clinicians in recruiting the appropriate candidates for clinical trials. In a previous study, Xing et al (12) revealed that the Advanced Liver Cancer Prognostic System (ALCPS) was the optimal staging system for predicting 3-month and overall survival rates among 12 systems analyzed. However, ALCPS is too complicated for clinical use. The ALCPS was based on 11 prognostic factors with different weights: Ascites; abdominal pain; weight loss; Child-Pugh class; alkaline phosphatase; total bilirubin; $\alpha$-fetoprotein (AFP); urea; portal vein thrombosis; tumor size; and presence of lung metastases (16). HCC patients were scored and classified into 3 groups: Good risk (0-8); intermediate risk (9-15); and poor risk $(>15)$. It is difficult to keep in mind all the factors and the scores. Zhang et al (13) suggested that CUPI was the optimal staging system in predicting survival in patients with unresectable HCC. In the study by Zhang et al, $89 \%$ of subjects were infected with $\mathrm{HBV}, 3 \%$ of subjects were infected with $\mathrm{HCV}$ and $2 \%$ of subjects developed HCC due to alcohol (13). As no consensus currently exists, the present study aimed to identify the best staging system for use in clinical trials in China, by restricting the subjects to only patients with $\mathrm{HBV}$-associated advanced HCC without any antitumor therapy. The results of the present study will assist patients and their doctors to gain insight into the natural history of untreated HCC, which will aid clinicians in making decisions of whether to accept other palliative treatments.

In the present study, the ability of seven different staging systems to predict 3- and 6-month and cumulative survival rates of patients with advanced $\mathrm{HBV}$-associated $\mathrm{HCC}$ was evaluated. This large cohort of patients from The Third Affiliated Hospital of Sun Yat-sen University (SYSU) in Guangzhou, China, will assist in establishing the inclusion criteria for clinical trials in China.

\section{Patients and methods}

Ethical approval. The present study was reviewed and approved by The Third Affiliated Hospital of SYSU Institutional Review Board. All study participants, or their legal guardian, provided written informed consent prior to study enrollment. The technical appendix, statistical code and full datasets are available from the corresponding author. Participants provided informed consent for data sharing. No additional data are available.

Patients. A total of 220 consecutive patients diagnosed with advanced HBV-associated HCC between September 2008 and June 2010 at The Third Affiliated Hospital of SYSU were retrospectively collected and analyzed. Patients were included if they were diagnosed with HCC and were hepatitis B surface antigen-positive. According to American Association for the study of Liver diseases guidelines (17), the diagnosis of HCC was confirmed by biopsy, or based on the non-invasive criteria which is only applicable to cirrhotic patients: In nodules $>2 \mathrm{~cm}$, one imaging technique demonstrating arterial hypervascularity and venous/late phase washout. In nodules of between 1 and $2 \mathrm{~cm}$, two techniques with a high coincidence [computed tomography (CT), contrast-enhanced ultrasound and magnetic resonance imaging (MRI)] are required (17). Patients diagnosed with advanced HBV-associated HCC belong to a heterogeneous group with marked differences in median survival ranges, from 1 month to $>1.5$ years. Patients included in the present study belonged to Barcelona Clinic Liver Cancer (BCLC) staging for hepatocellular carcinoma stages C and D (17). Specific patients with BCLC C and D stage HCC in The Third Affiliated Hospital of SYSU also received standard antitumor therapy and were excluded from the present study, in order to study the natural history of advanced HCC. Patients included in the present study did not receive any standard antitumor therapy. The majority of patients with Cancer of the Liver Italian Program (CLIP) 0, Okuda 0, Japan Integrated Staging (JIS) 0 or 1 HCC exhibited treatable disease, but did not receive antitumor treatment, partially due to lack of insurance coverage. Certain patients rejected antitumor therapy, but received the best supportive care and traditional Chinese herbal medicine. In the present study, 82 patients were classified with BCLC D HCC. A total of 24 patients survived $>3$ months, and these patients exhibited improved prognosis compared with the other 58 patients, who survived $<3$ months. Certain patients were classified with BCLC D HCC due to a diagnosis of performance status (PS) 3-4 (18) at first diagnosis and following the best supportive care, PS may improve. Patients with BCLC D were a mixed group with different prognoses. 
In the baseline evaluation, laboratory studies, imaging studies (CT or MRI) and clinical examination were required. Data were collected once the patients were diagnosed with advanced $\mathrm{HBV}$-associated $\mathrm{HCC}$. The survival time was defined as the time from diagnosed date to the date of mortality or last contact with surviving patients.

Standard antitumor therapy refers to therapies recommended by National Comprehensive Cancer Network guidelines, including transplantation, surgery, transarterial chemoembolization or percutaneous ethanol injection, chemotherapy or targeted therapy (19). The patients in the present study did not receive standard antitumor therapy as they were not suitable for treatment, or they declined all therapeutic options.

Patients lacking data required for any of the studied staging systems or with prior malignancies or secondary cancers were excluded. If they were lost to follow-up within 3 months of diagnosis, they were also excluded.

Data collection. Data acquisition from the electronic charts was approved by the institutional ethics committee. Baseline data were collected to classify patients according to the CLIP, CUPI, tumor-node-metastasis (TNM), JIS, Okuda, China Integrated Score (CIS) and BCLC systems.

JIS scores may be original or modified (11). In modified JIS scores, the ICGR15 value is used instead of hepatic encephalopathy, and the threshold levels for prothrombin time is different from the Child-Pugh score. As the indocyanine green retention test value is not routinely examined in The Third Affiliated Hospital of SYSU, original JIS scores were used in the present study. Original JIS scores used the Child-Pugh score.

A range of demographic data was collected, including risk factors, clinical data including performance status, imaging data and liver cirrhosis manifestations.

In particular, the following variables were retrieved for the analysis of the present study: Gender, age, date of diagnoses and date of mortality or last follow-up, the general condition of the patients (abdominal pain, weight loss). Performance status (PS) was retrospectively assessed from the clinical records (18). Tumor characteristics were assessed via CT or MRI, including the diameter of the largest lesion, the number of lesions, organ invasion, lymph node status and portal vein thrombosis. Laboratory data were also collected, including routine blood tests, liver function tests [total bilirubin, albumin and $\gamma$-glutamyltranspeptidase (GGT)], alkaline phosphatase (ALP), aspartate aminotransferase (AST), AFP levels, blood urea nitrogen (BUN) and coagulation function (prothrombin time/international normalized ratio values). A chest X-ray was routinely performed for distant metastases. A brain CT was performed if they had paralysis or terrible headache. Bone Emission Computed Tomography was performed if they had bone pain or bone mass.

Statistical analysis. The main endpoints of the present study were 3-month survival and overall survival. The secondary endpoint was 6-month survival. Kaplan-Meier estimator survival curves and log-rank tests were used to compare overall survival rate predicted using different staging systems.

Different staging systems consist of a different number of stages. For example, CLIP includes 7 stages, JIS and CIS include 6 stages, and Okuda and CUPI include only 3 stages. Likelihood ratio tests (LRTs) were used to compare different staging systems. The degree of freedom was set at 1 , such that different prognostic systems with different stages were able to be compared. Akaike information criterion (AIC) values were also used to avoid statistical bias. If the likelihood ratio value of a staging system is higher and AIC value is lower compared with other systems, it was considered to have an improved prognostic ability.

Cox's proportional hazard model was used to identify independent predictive variables of survival. Statistically significant prognostic variables were identified in univariate analyses. Subsequently, these variables were included in multivariate analyses.

The sensitivity and specificity of the predictive value each staging system for 3- and 6-month mortality were analyzed by relative operating characteristic (ROC) curve analysis, and the DeLong et al (12) test was used to compare the area under curve (AUC) of the ROC curves. A larger area indicated an improved discriminatory ability in stratifying patients with different outcomes.

All the important prognostic factors for advanced HCC were included in the univariate and multivariate analyses by Cox's proportional hazard model. These factors included sex, age, HBV status, PS, AFP, albumin, bilirubin, ALP, hemoglobin, hepatic encephalopathy, fibrinogen, prothrombin time, ascites, weight loss, abdominal pain, vascular involvement, tumor size, lymph node status, metastases and Child-Pugh score.

All the analyses of the data were performed using SPSS (version 20.0; IBM SPSS, Armonk, NY, USA), STATA (version 12.0; StataCorp LLC, College Station, TX, USA) and SAS (version 9.1.3; SAS Institute, Inc., Cary, NC, USA).

\section{Results}

Patient characteristics. All 220 patients included in the present study did not receive antitumor treatment and were of Chinese ethnicity. Patients were staged using CLIP, CUPI, TNM, JIS, Okuda, CIS and BCLC systems. All authors were familiar with the seven staging systems. Table I summarizes the baseline characteristics of all patients. The patients included $198(90 \%)$ males and $22(10 \%)$ females. The median age of the patients was 55.7 (range, 19-85 years). The 90-day survival rate was $45.5 \%$ and the 6-month survival rate was $30.5 \%$. All patients included were $\mathrm{HBV}$ carriers. Of note, $57.7 \%$ of the patients were stage III and $20.9 \%$ of the patients were stage IV, based on TNM seventh edition staging (19). A total of $28.6 \%$ of the patients were Child-Pugh class C.

Evaluation of staging systems. Kaplan-Meier estimator curves were generated using SPSS software for CLIP (Fig. 1), CIS (Fig. 2), CUPI (Fig. 3), Okuda (Fig. 4), TNM (Fig. 5), JIS (Fig. 6) and BCLC (Fig. 7). Significant statistical differences were observed between different prognostic strata for CLIP, CUPI, TNM, JIS, Okuda, CIS and BCLC ( $\mathrm{P}<0.05$ in all cases, log-rank test).

The LRT and AIC results are listed in Table II. The LRT $\chi^{2}$ values for CLIP, CIS, CUPI, OCUDA, TNM, JIS and BCLC were 75.6, 48.4, 46.7, 36.0, 21.0, 46.8 and 7.24, respectively. The AIC values for CLIP, CIS, CUPI, OCUDA, TNM, JIS and 
Table I. Baseline demographic characteristics of study participants.

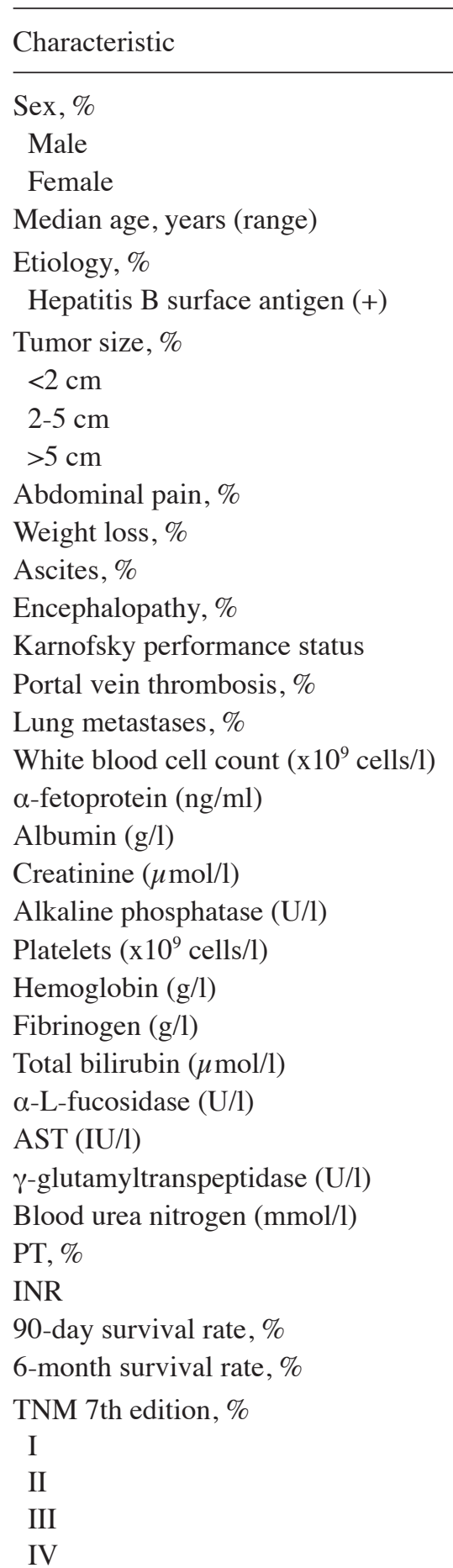

Child-Pugh class, $\%$

$$
\begin{aligned}
& \text { A } \\
& \text { B } \\
& \text { C }
\end{aligned}
$$

CUPI, \%

$$
\begin{aligned}
& 0 \\
& 1
\end{aligned}
$$$$
2
$$

Okuda, \%

$$
\text { I }
$$$$
\text { II }
$$

Patients $(n=220)$

$90(198 / 220)$
$10(22 / 220)$
$55.7(19-85)$
$100(220 / 220)$

$8.2(18 / 220)$
$14.1(31 / 220)$
$77.7(171 / 220)$
$43.6(96 / 220)$
$39.1(86 / 220)$

$58.6(129 / 220)$

$2.3(5 / 220)$

70 (20-90)

$68.2(150 / 220)$

$14.1(31 / 220)$

$6.2(1.84-25.09)$

$668.8(1.0-21620.0)$

34.45 (13.6-51.8)

$68.77(29.0-277.0)$

$145.5(33.0-767.0)$

$137.0(4-503)$

119.5 (56.0-177.0)

$2.91(0.9-9.4)$

35.88 (4.69-864.86)

$27.0(4.0-124.0)$

$141.0(17.0-2218.0)$

$177.0(17.0-1128.0)$

$5.27(1.63-25.42)$

$76(31-100 \%)$

$1.19(0.85-2.53)$

$45.5(100 / 220)$

$30.5(67 / 220)$

$11.8(26 / 220)$

$9.5(21 / 220)$

$57.7(127 / 220)$

$20.9(46 / 220)$

$25.5(56 / 220)$

$45.9(101 / 220)$

$28.6(63 / 220)$

$19.5(43 / 220)$

$48.2(106 / 220)$

\begin{tabular}{|c|c|}
\hline Characteristic & Patients $(n=220)$ \\
\hline \multicolumn{2}{|l|}{ CLIP, \% } \\
\hline 0 & $2.3(5 / 220)$ \\
\hline 1 & $9.5(21 / 220)$ \\
\hline 2 & $14.1(31 / 220)$ \\
\hline 3 & $16.4(36 / 220)$ \\
\hline 4 & $22.7(50 / 220)$ \\
\hline 5 & $25.9(57 / 220)$ \\
\hline 6 & $9.1(20 / 220)$ \\
\hline \multicolumn{2}{|l|}{ BCLC, $\%$} \\
\hline A & 0 \\
\hline B & 0 \\
\hline $\mathrm{C}$ & $62.7(138 / 220)$ \\
\hline $\mathrm{D}$ & $37.3(82 / 220)$ \\
\hline \multicolumn{2}{|l|}{ JIS, \% } \\
\hline 0 & $0.5(1 / 220)$ \\
\hline 1 & $5.9(13 / 220)$ \\
\hline 2 & $13.6(30 / 220)$ \\
\hline 3 & $25.5(56 / 220)$ \\
\hline 4 & $37.7(83 / 220)$ \\
\hline 5 & $16.8(37 / 220)$ \\
\hline \multicolumn{2}{|l|}{ CIS, $\%$} \\
\hline 0 & $10.5(23 / 220)$ \\
\hline 1 & $26.8(59 / 220)$ \\
\hline 2 & $33.6(74 / 220)$ \\
\hline 3 & $17.7(39 / 220)$ \\
\hline 4 & $6.8(15 / 220)$ \\
\hline 5 & $4.5(10 / 220)$ \\
\hline
\end{tabular}

$32.3(71 / 220)$

$15.5(34 / 220)$

$54.1(119 / 220)$

$30.5(67 / 220)$
Table I. Continued.

AST, aspartate aminotransferase; PT, prothrombin time; INR, international normalized ratio; tumor-node-metastasis; CUPI, Chinese University Prognostic Index; CLIP, Cancer of the Liver Italian Program; BCLC, Barcelona Clinic Liver Cancer; JIS, Japan Integrated Staging; CIS, China Integrated Score.

BCLC were 1601.5, 1632.3, 1629.9, 1641.1, 1654.8, 1627.4 and 1671.1, respectively. CLIP exhibited the highest $\chi^{2}$ value and lowest AIC value.

Pairwise comparison of the AUC of ROC curves predicting 3-month survival revealed that CLIP ranked highest, followed by CIS. There was no significant difference between CLIP and CIS observed when predicting 3-month survival (Table III). In terms of predicting 6-month survival, the AUC of CLIP ranked first, followed by JIS. There was a significant difference between CLIP and JIS (Table IV).

Comparison of the predictive ability of the seven staging systems for 3- and 6-month survival was calculated by ROC analysis. The AUC curves of the seven staging systems for predicting 3- and 6-month survival are demonstrated in Figs. 8 and 9.

When predicting 3-month survival, the AUCs of CLIP, CIS, CUPI, Okuda, TNM, JIS and BCLC were 0.806, 0.772, $0.751,0.731,0.643,0.754$ and 0.622 , respectively. When 

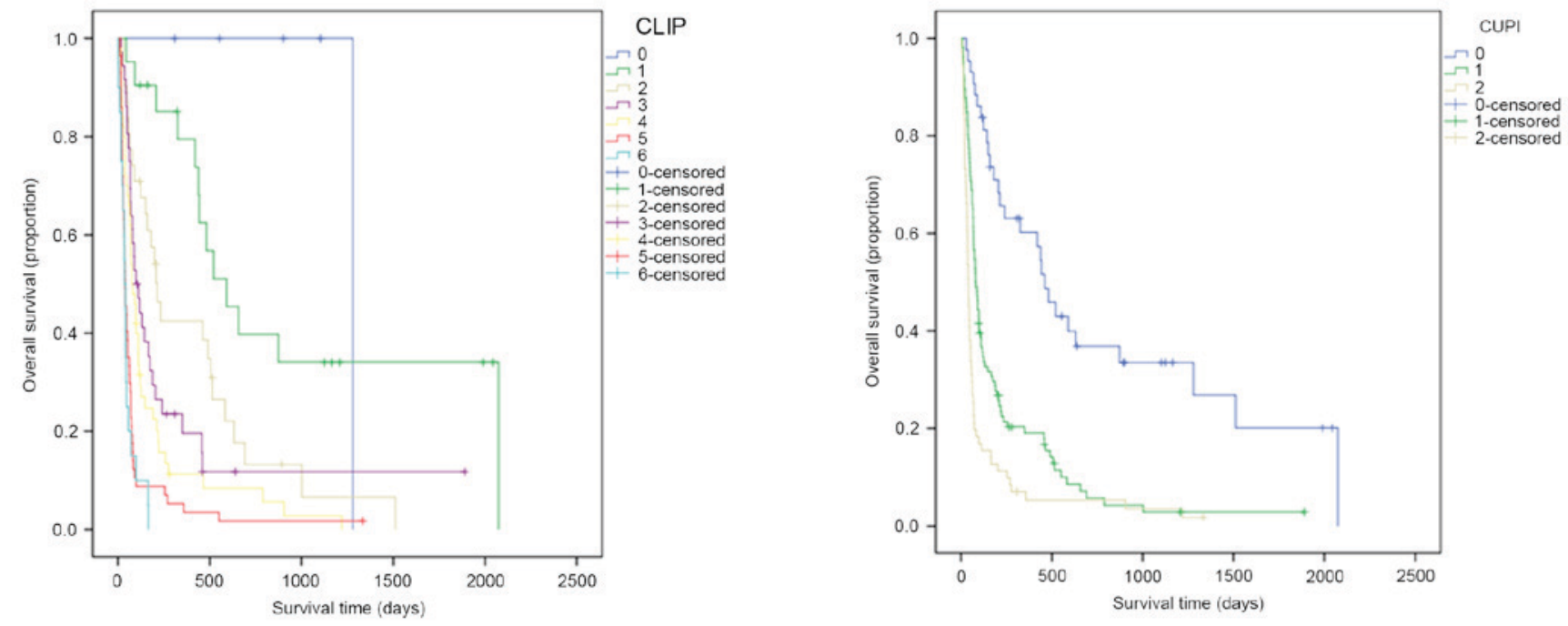

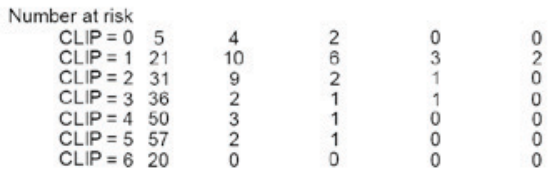

Figure 1. Kaplan-Meier estimator survival curves for patients with advanced hepatocellular carcinoma staged by CLIP. CLIP, the Cancer of the Liver Italian Program.

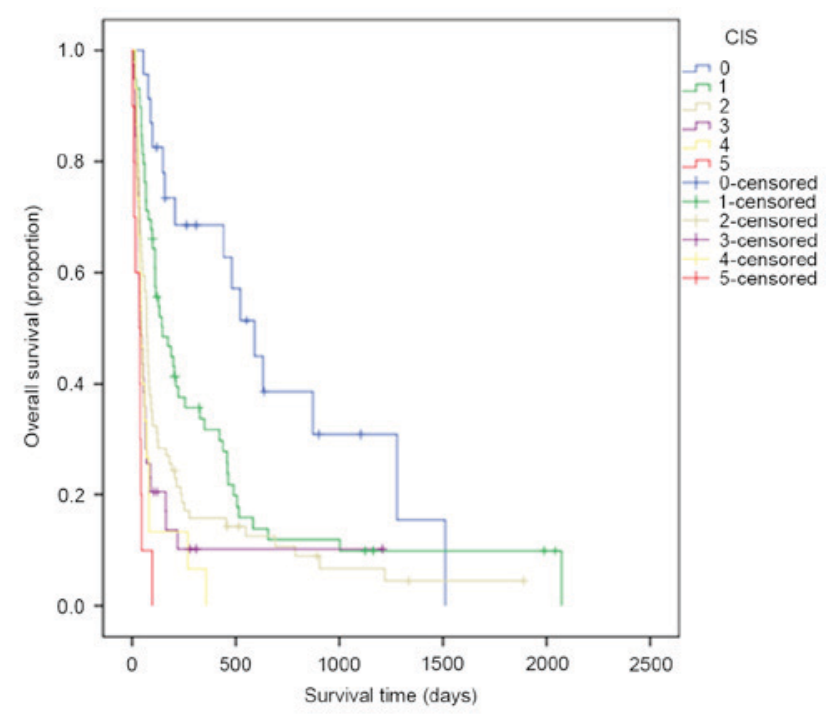

$\begin{array}{rrrrrr}\text { Number at risk } & & & & & \\ \mathrm{CIS}=0 & 23 & 10 & 3 & 1 & 0 \\ \mathrm{CIS}=1 & 59 & 10 & 6 & 3 & 2 \\ \mathrm{CIS}=2 & 74 & 9 & 3 & 1 & 0 \\ \mathrm{CIS}=3 & 39 & 1 & 1 & 0 & 0 \\ \mathrm{CIS}=4 & 15 & 0 & 0 & 0 & 0 \\ \mathrm{CIS}=5 & 10 & 0 & 0 & 0 & 0\end{array}$

Figure 2. Kaplan-Meier estimator survival curves for patients with advanced hepatocellular carcinoma staged by CIS. CIS, China Integrated Score.

predicting 6-month survival, the AUCs of CLIP, CIS, CUPI, Okuda, TNM, JIS and BCLC were 0.828, 0.729, 0.717, 0.692, $0.664,0.746$ and 0.575 , respectively.

Prognostic factors of survival. Independent prognostic factors for overall survival were identified using univariate and multivariate analyses. These factors are summarized in Table V. Univariate analyses identified age, metastasis, PS,

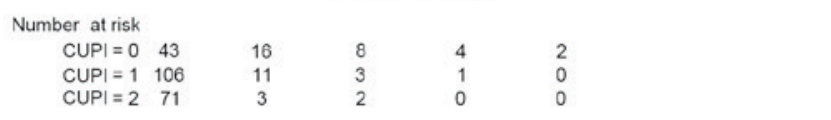

Figure 3. Kaplan-Meier estimator survival curves for patients with advanced hepatocellular carcinoma staged by CUPI. CUPI, Chinese University Prognostic Index.

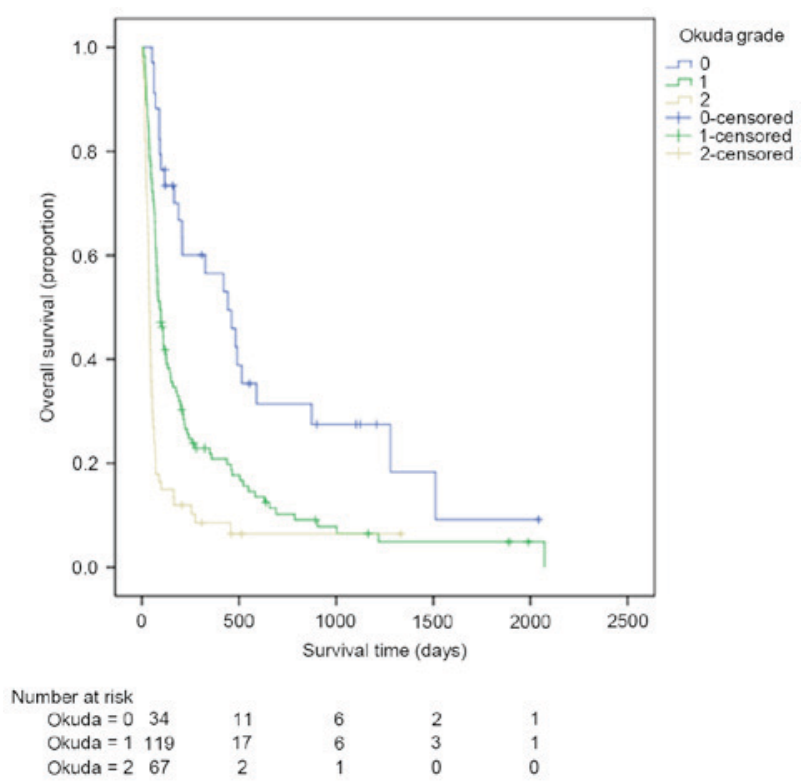

Figure 4. Kaplan-Meier estimator survival curves for patients with advanced hepatocellular carcinoma staged by Okuda.

tumor size, weight loss, ALP, AFP, albumin, BUN, creatinine, $\alpha$-L-fucosidase, platelet count, GGT, WBC, neutrophil count, fibrinogen, portal vein thrombus and Child-Pugh class as independent predictors of overall survival. Multivariate analyses identified tumor size, AFP, BUN, portal vein thrombus, Child-Pugh class and neutrophil count as independent prognostic factors.

\section{Discussion}

The majority of patients are diagnosed with advanced $\mathrm{HCC}$ at their initial diagnosis and a number of them are 


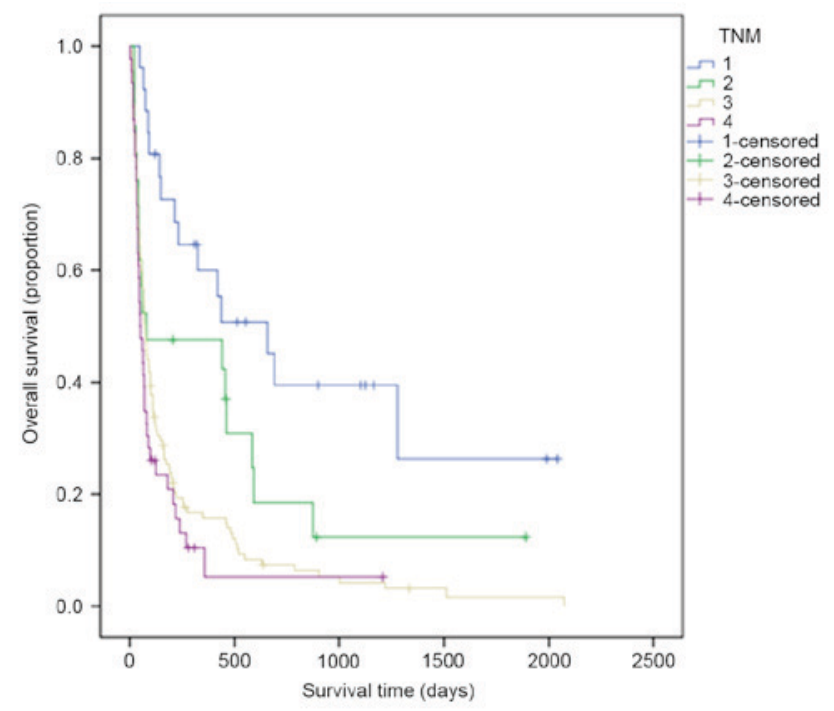

Number at risk

$\begin{array}{lccccc}\text { TNM }=1 & 26 & 11 & 6 & 2 & 1 \\ \text { TNM }=2 & 21 & 5 & 1 & 1 & 0 \\ \text { TNM }=3 & 127 & 13 & 5 & 2 & 1 \\ \text { TNM }=4 & 46 & 1 & 1 & 0 & 0\end{array}$

Figure 5. Kaplan-Meier estimator survival curves for patients with advanced hepatocellular carcinoma staged by TNM. TNM, tumor-node-metastasis.
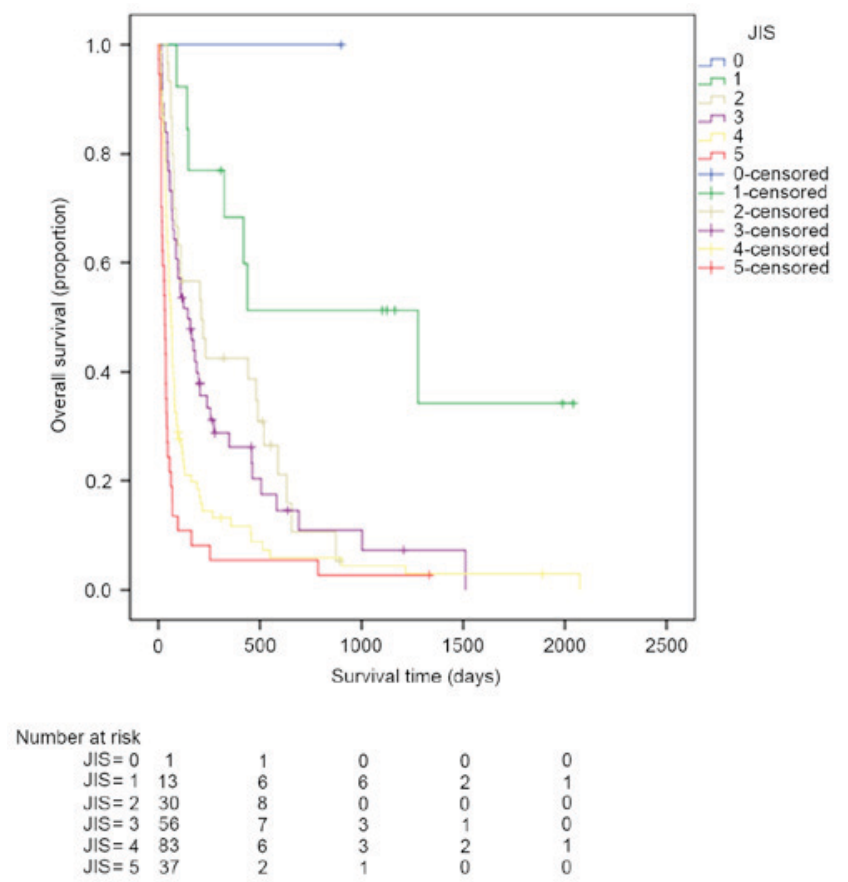

$\begin{array}{ll}0 & 0 \\ 2 & 1 \\ 0 & 0 \\ 1 & 0 \\ 2 & 1 \\ 0 & 0\end{array}$

Figure 6. Kaplan-Meier estimator survival curves for patients with advanced hepatocellular carcinoma staged by JIS. JIS, Japan Integrated Staging.

unresectable (17). In these patients, the therapeutic option is systemic therapy (20). In the Sorafenib Hepatocellular Carcinoma Assessment Randomized Protocol study (4), sorafenib was demonstrated to significantly prolong median overall survival of patients with advanced HCC (sorafenib arm, 10.7 months vs. placebo arm, 7.9 months; $\mathrm{P}<0.001)$ and the Asia-Pacific study (5) (sorafenib arm, 6.5 months vs. placebo arm, 4.2 months; $\mathrm{P}<0.01)$. Kaplan-Meier estimator survival curves of HCC begin to deviate from the third month
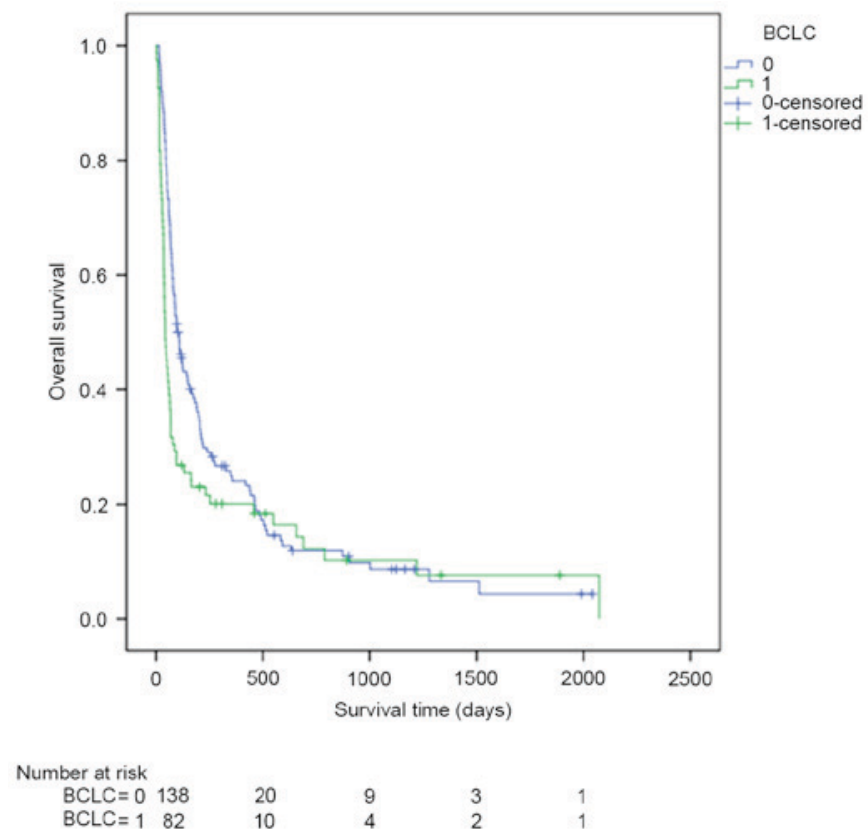

Figure 7. Kaplan-Meier estimator survival curves for patients with advanced hepatocellular carcinoma staged by BCLC. BCLC, Barcelona Clinic Liver Cancer.

subsequent to patients receiving sorafenib $(4,5)$. Life expectancy $>3$ months is an essential inclusion criterion of clinical trials. A previous study had assessed several staging systems for HCC (21), including CLIP, CUPI, JIS, BCLC, CIS, TNM and Okuda. There is currently no consensus on which staging system ranks first for predicting 3- and 6-month and overall survival rates in patients with advanced $\mathrm{HBV}$-associated $\mathrm{HCC}$.

In the present study, the seven current staging systems were evaluated in order to identify out the most suitable staging system. In the selected patient population of the present study, the CLIP staging system was the most capable of predicting 3- and 6-month mortality and overall survival rates in patients with advanced HBV-associated HCC. The TNM staging system included only anatomical factors, and did not perform well in overall survival prediction. Only patients with BCLC C or D $\mathrm{HCC}$ were recruited in the present study, as all patients with BCLC A and BCLC B HCC received specific antitumor therapies and were excluded. For this reason, BCLC did not perform well and demonstrated poor discriminatory ability: It exhibited the lowest likelihood ratio value and the highest AIC value.

CLIP was the most accurate staging system in predicting survival rates in the present study. CLIP is a frequently used staging system in stratifying patients with HCC whose main etiology is hepatitis C (10). The CLIP system scores patients from 0 to 6 on the basis of four factors: Portal vein thrombosis, tumor morphology, AFP level and Child-Pugh class (10): Higher scores predict poorer outcomes. Anatomical factors and liver function were assessed in CLIP. A previous retrospective study demonstrated that CLIP exhibited a good ability to predict recurrence following curative resection in 174 patients with $\mathrm{HCC}$ with hepatitis B as the main etiology (22). In the present study of patients with HBV-associated HCC exclusively, CLIP also exhibited the best predictive ability with regard to survival outcomes. 
Table II. Homogeneity LRT $\chi^{2}$ test and AIC of various hepatocellular carcinoma staging systems.

\begin{tabular}{lccc}
\hline Staging system & Homogeneity LRT $\chi^{2}$ test & AIC & P-value \\
\hline Cancer of the Liver Italian Program & 70.6 & 1601.5 & $<0.0001$ \\
China Integrated Score & 48.4 & 1632.3 & $<0.0001$ \\
Chinese University Prognostic Index & 46.7 & 1629.9 & $<0.0001$ \\
Okuda & 36.0 & 1641.1 & $<0.0001$ \\
Tumor-node-metastasis & 21.0 & 1654.8 & 1627.4 \\
Japan Integrated Staging & 46.8 & 1671.1 & $<0.0001$ \\
Barcelona Clinic Liver Cancer & 7.24 & 0.0001
\end{tabular}

LRT, likelihood ratio test; AIC, Akaike information criterion.

Table III. Pairwise comparison of relative operating characteristic curves predicting 3-month survival.

\begin{tabular}{lllr}
\hline System & System & Difference & P-value \\
\hline CLIP & CIS & 0.0346 & 0.2001 \\
CLIP & JIS & 0.0526 & 0.0320 \\
CLIP & CUPI & 0.0559 & 0.0312 \\
CLIP & Okuda & 0.0757 & 0.0110 \\
CLIP & TNM & 0.164 & $<0.0001$ \\
CLIP & BCLC & 0.185 & $<0.0001$ \\
CIS & JIS & 0.0180 & 0.5367 \\
CIS & CUPI & 0.0213 & 0.4983 \\
CIS & Okuda & 0.0410 & 0.2045 \\
CIS & TNM & 0.129 & 0.0001 \\
CIS & BCLC & 0.150 & $<0.0001$ \\
JIS & CUPI & 0.00333 & 0.9147 \\
JIS & Okuda & 0.0231 & 0.4718 \\
JIS & TNM & 0.111 & 0.0011 \\
JIS & BCLC & 0.132 & 0.0001 \\
CLIP & CIS & 0.0346 & 0.2001 \\
CLIP & JIS & 0.0526 & 0.0320 \\
\hline CLIP, Cancer of the Liver Italian Program; CUPI, Chinese University \\
Prognostic & Index $;$ TNM, tumor-node-metastasis; & JIS, Japan \\
Integrated & Staging; BCLC, Barcelona Clinic Liver Cancer; CIS, \\
China Integrated Score. & & \\
\hline
\end{tabular}

The results of the present study also suggested that CLIP is capable of stratifying patients with HCC with HBV. The utility of CLIP may be additionally improved: A previous study suggested that CLIP may have an improved discriminatory ability by adding abdominal pain, presence of esophageal varices, PS and AST into the staging system (23). This requires prospective validation.

In the present study, when predicting 3-month mortality, the prognostic values of CIS ranks the second following CLIP. In 2010, Zhang et al proposed a HCC staging system termed the CIS (14). CIS included three factors: adjusted TNM stage, Child-Pugh class and AFP. The score of adjusted TNM stage was defined as follows: 0, TNM stage I-III comprising
Table IV. Pairwise comparison of receiver operating characteristic curves predicting 6-month survival.

\begin{tabular}{lllr}
\hline System & System & Difference & P-value \\
\hline CLIP & CIS & 0.0996 & 0.0007 \\
CLIP & JIS & 0.0826 & 0.0010 \\
CLIP & CUPI & 0.111 & $<0.0001$ \\
CLIP & Okuda & 0.136 & $<0.0001$ \\
CLIP & TNM & 0.164 & $<0.0001$ \\
CLIP & BCLC & 0.254 & $<0.0001$ \\
CIS & JIS & 0.0170 & 0.6087 \\
CIS & CUPI & 0.0115 & 0.7274 \\
CIS & Okuda & 0.0368 & 0.2942 \\
CIS & TNM & 0.0646 & 0.0837 \\
CIS & BCLC & 0.154 & $<0.0001$ \\
JIS & CUPI & 0.0285 & 0.3518 \\
JIS & Okuda & 0.0538 & 0.1320 \\
JIS & TNM & 0.0816 & 0.0294 \\
JIS & BCLC & 0.171 & $<0.0001$ \\
CLIP & CIS & 0.0996 & 0.0007 \\
CLIP & JIS & 0.0826 & 0.0010 \\
& & &
\end{tabular}

CLIP, Cancer of the Liver Italian Program; CUPI, Chinese University Prognostic Index; TNM, tumor-node-metastasis; JIS, Japan Integrated Staging; BCLC, Barcelona Clinic Liver Cancer; CIS, China Integrated Score.

uninodular tumor or multinodular tumors, in a single lobe; 1 , TNM stage IVa, multinodular tumors within multiple lobes, peritoneum invasion or involves veins; 2 , stage IVb HCC that exhibits distant metastases. The score of the Child-Pugh class was defined as follows: 0, Child-Pugh class A; 1, Child-Pugh class B; 2, Child-Pugh class C. The score of AFP was defined as follows: $0, \leq 400 \mathrm{mg} / 1 ; 1,>400 \mathrm{mg} / \mathrm{l}$. The score of each factor was summed and a CIS score was calculated. CIS is easy to use (14). In the study by Zhang et al a number of staging systems were compared by analyzing 196 patients with HCC who received non-surgical treatment, and it was concluded that CIS was not good in predicting survival in this specific cohort of patients (13). Additional studies are required to 
Table V. Independent prognostic factors for overall survival in patients with advanced hepatocellular carcinoma.

\begin{tabular}{lccc}
\hline Covariate & Hazard ratio for mortality & 95\% confidence interval of Exp(b) & P-value \\
\hline$\alpha$-fetoprotein & 0.56 & $0.37-0.87$ & 0.01 \\
Blood urea nitrogen & 3.23 & $2.02-5.17$ & $<0.01$ \\
Child-Pugh class of original Child-Pugh system & 1.36 & $1.07-1.732$ & 0.01 \\
Neutrophil count & 1.29 & $1.03-1.62$ & 0.03 \\
Portal vein thrombosis & 0.62 & $0.38-0.99$ & 0.05 \\
Tumor size & 0.57 & $0.37-0.87$ & 0.01 \\
\hline
\end{tabular}

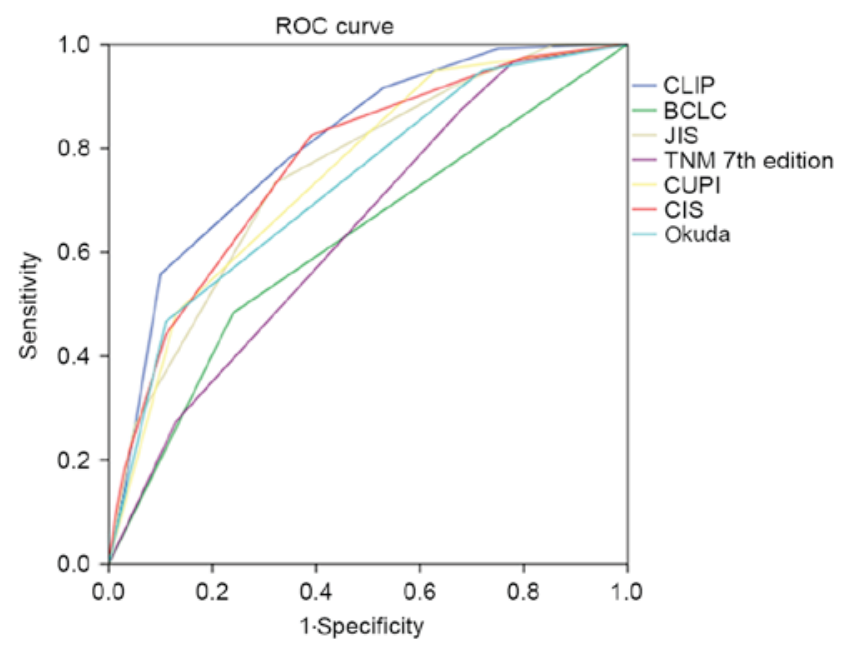

Figure 8. ROC curves of CLIP, CIS, CUPI, Okuda, TNM, JIS and BCLC when predicting 3-month survival. ROC, relative operating characteristics; CLIP, the Cancer of the Liver Italian Program; CIS, China Integrated Score; CUPI, Chinese University Prognostic Index; TNM, tumor-node-metastasis; JIS, Japan Integrated Staging; BCLC, Barcelona Clinic Liver Cancer.

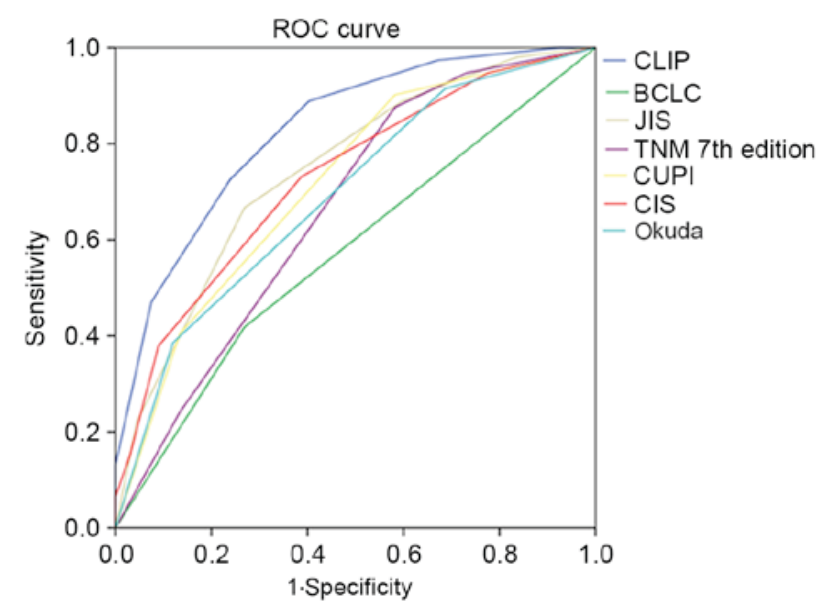

Figure 9. ROC curves of CLIP, CIS, CUPI, Okuda, TNM, JIS and BCLC when predicting 6-month survival. ROC, relative operating characteristics; CLIP, the Cancer of the Liver Italian Program; CIS, China Integrated Score; CUPI, Chinese University Prognostic Index; TNM, tumor-node-metastasis; JIS, Japan Integrated Staging; BCLC, Barcelona Clinic Liver Cancer.

confirm whether CIS will perform well in predicting 3-month mortality of patients with HCC who did not receive any standard anticancer treatment.
In the present study, when predicting 6-month mortality, the prognostic values of JIS ranks second to CLIP. JIS score was proposed based on analysis of 722 Japanese patients with HCC (11). Child-Pugh class defined by original Child-Pugh system and TNM stage by the Liver Cancer Study Group of Japan criteria were incorporated into the JIS score in a previous study (11). Kudo et al (24) demonstrated that in patients with early-stage HCC, JIS demonstrated a higher predictive value for survival compared with CLIP. In the present study, in patients with advanced HCC, the predictive value of JIS was lower compared with that of CLIP. In the present study, one patient scored JIS 0 and 13 patients scored JIS 1 . The PS of the patients with JIS 0 and 1 scores was 1 , and they were classified as BCLC C. JIS did not incorporate PS into the system. Previously, Nishikawa et al (25) proposed a system in which performance status was combined with the Japan Integrated Staging system (PS-JIS), and PS-JIS was compared with four other prognostic systems, including the original JIS system, BCLC, TNM and CLIP scoring systems. It was demonstrated that for all patients, at each time point (1-, 3- and 5-year time points), the c-index of the PS-JIS system was the highest among five staging systems, indicating that the PS-JIS system possessed the best predictability among these staging systems (25). Another study suggested that incorporating AFP into JIS improved its prognostic ability (26). However, the modification of JIS was not widely accepted due to lack of validation.

Different studies have revealed differential ranking of the various staging systems, and the best staging system for HCC remains unknown. To the best of our knowledge, the present study is the first to focus only on patients with HBV-associated advanced HCC who did not receive any standard antitumor therapy. The present study indicated that Child-Pugh class defined by original Child-Pugh system BUN, AFP, portal vein thrombosis, tumor size and neutrophil count were predictors of survival in the cohort of patients with HCC. The results of the present study revealed again that the ranking of staging system may vary, and prognostic factors may vary for different systems of HCC patients (27).

Patients with advanced HCC are a heterogeneous group. It is difficult to construct a standard staging system that is able to precisely predict survival. A number of confounding factors should be taken into account, including anatomic factors, liver function, serum tumor markers, symptoms and comorbidities. The construction of a universally accepted HCC staging system appears to be unachievable. A large number of Asian and Caucasian patients with HCC should be recruited to 
perform careful statistical analyses and prospective validation The present study was a retrospective study, and all patients were from a single hospital. The next step is to evaluate the hypothesis of the present study prospectively in patients with HCC from different institutes and different countries, and in patients with $\mathrm{HCC}$ with $\mathrm{HBV}, \mathrm{HCV}$ and alcohol-associated risk factors.

Staging classifications continue to be refined by ongoing studies. In the future, tumor markers may be incorporated into staging systems to improve prognostic ability. A number of tumor markers prognostic of survival have been identified. The BALAD score is a novel scoring system based on five serum markers: Bilirubin; albumin; lens culinaris agglutinin-reactive AFP, AFP and des- $\gamma$-carboxy prothrombin, and thus, it is termed the BALAD score (28). The BALAD score is easy to use and stage can be evaluated with the use of only one serum sample (28). Molecular markers including Wiskott-Aldrich syndrome protein family member 3, hepatocellular carcinoma-associated protein JCL-1, DEP domain-containing protein 1A, prolyl hydroxylase domain-containing protein 2, microRNA (miR)-9, miR-24-3p, miR-148a and keratin 19 (KRT19) have an implied association with survival outcome (29-36). Kaseb et al (37) integrated plasma vascular endothelial growth factor into CLIP and constructed V-CLIP to stratify patients. They concluded that V-CLIP exhibited improved discriminatory ability compared with CLIP $(\mathrm{P}=0.05)$. A previous study identified five genes (TATA box-binding protein-associated factor F9, receptor activity-modifying protein 3, hematological and neurological expressed 1, KRT19 and Ras-related nuclear protein) with prognostic value: Patients were stratified with $\mathrm{HCC}$ to be good risk group and poor risk group by using a gene expression score (38). Integrating these tumor markers into current staging systems may assist in additionally improving the sensitivity and specificity of these staging classifications. In the selected group of patients with advanced HBV-associated HCC in the present study, CLIP was identified to be the best staging system for predicting 3and 6-month and cumulative survival rates. CIS was adequate for predicting 3-month mortality and JIS was adequate for predicting 6-month mortality. Prospective and multicenter validations are required to determine which staging system is best.

\section{Acknowledgements}

The statistical methods used in the present study were reviewed by FanSong Zeng from the School of Public Health, Sun Yat-sen University (Guangzhou, China). The present study was supported by grants from the National Natural Science Foundation of China (grant nos. 31600710 and 81372374), the Combination Project of Production, the scientific research project for University belong to Guangzhou Municipal (grant no. 1201630019), and Natural Science Foundation of Guangdong (grant nos. 2014A030313146 and 2016A030313302).

\section{References}

1. Torre LA, Bray F, Siegel RL, Ferlay J, Lortet-Tieulent J and Jemal A: Global cancer statistics, 2012. CA Cancer J Clin 65: $87-108,2015$
2. Chen W, Zheng R, Baade PD, Zhang S, Zeng H, Bray F, Jemal A, Yu XQ and He J: Cancer statistics in China, 2015. CA Cancer J Clin 66: 115-132, 2016.

3. Lai CL, Ratziu V, Yuen MF and Poynard T: Viral hepatitis B. Lancet 362: 2089-2094, 2003.

4. Llovet JM, Ricci S, Mazzaferro V, Hilgard P, Gane E, Blanc JF, de Oliveira AC, Sanoro A, Raoul JL, Forner A, et al: Sorafenib in advanced hepatocellular carcinoma. N Engl J Med 359: 378-390, 2008.

5. Cheng AL, Kang YK, Chen Z, Tsao CJ, Qin S, Kim JS, Luo R, Feng J, Ye S, Yang TS, et al: Efficacy and safety of sorafenib in patients in the Asia-Pacific region with advanced hepatocellular carcinoma: A phase III randomised, double-blind, placebo controlled trial. Lancet Oncol 10: 25-34, 2009.

6. Bruix J, Gores GJ and Mazzaferro V: Hepatocellular carcinoma: Clinical frontiers and perspectives. Gut 63: 844-855, 2014.

7. Bertino G, Demma S, Ardiri A, Proiti M, Gruttadauria S, Toro A, Malaguarnera G, Bertino N, Malaguarnera M, Malaguarnera M and Di Carlo I: Hepatocellular carcinoma: Novel molecular targets in carcinogenesis for future therapies. Biomed Res Int 2014: 203693, 2014.

8. Abou-Alfa GK: Selection of patients with hepatocellular carcinoma for sorafenib. J Natl Compr Canc Netw 7: 397-403, 2009.

9. Abou-Alfa GK: Hepatocellular carcinoma: Molecular biology and therapy. Semin Oncol 33 (6 Suppl 11): S79-S83, 2006.

10. Llovet JM and Bruix J: Prospective validation of the cancer of the liver Italian program (CLIP) score: A new prognostic system for patients with cirrhosis and hepatocellular carcinoma. Hepatology 32: 679-680, 2000.

11. Kubo S, Tanaka H, Shuto T, Takemura S, Yamamoto T, Uenishi T, Tanaka S, Hai S, Yamamoto S, Ichikawa T, et al: Prognostic effects of causative virus in hepatocellular carcinoma according to the Japan integrated staging (JIS) score. J Gastroenterol 40: 972-979, 2005.

12. DeLong ER, DeLong DM and Clarke-Pearson DL: Comparing the areas under two or more correlated reciever operating characteristic curves: A nonparamentric approach. Biometrics 44: 837-845, 1988.

13. Zhang JF, Shu ZJ, Xie CY, Li Q, Jin XH, Gu W, Jiang FJ and Ling CQ: Prognosis of unresectable hepatocellular carcinoma: Comparison of seven staging systems (TNM, Okuda, BCLC, CLIP, CUPI, JIS, CIS) in a Chinese cohort. PLoS One 9: e88182, 2014.

14. Zhang BH, Wang XH, Yue HY and Ling CQ: A new staging system is more discriminant than conventional staging systems for unresectable hepatocellular carcinoma. J Cancer Res Clin Oncol 136: 821-827, 2010.

15. Adhoute X, Penaranda G, Raoul JL, Le Treut P, Bollon E, Hardwigsen $\mathrm{J}$, Castellani $\mathrm{P}$, Perrier $\mathrm{H}$ and Bourlière $\mathrm{M}$ : Usefulness of staging systems and prognostic scores for hepatocellular carcinoma treatments. World J Hepatol 8: 703-715, 2016.

16. Yau T, Yao TJ, Chan P, Ng K, Fan ST and Poon RT: A new prognostic score system in patients with advanced hepatocellular carcinoma not amendable to locoregional therapy: Implication for patient selection in systemic therapy trials. Cancer 113: 2742-2751, 2008.

17. Bruix J and Sherman M; Practice Guidelines Committee, American Association for the Study of Liver Diseases: Management of hepatocellular carcinoma. Hepatology 42: 1208-1236, 2005.

18. Oken MM, Creech RH, Tormey DC, Horton J, Davis TE, McFadden ET and Carbone PP: Toxicity and response criteria of the eastern cooperative oncology group. Am J Clin Oncol 5: 649-655, 1982 .

19. [Guideline] National Comprehensive Cancer Network. NCCN Hepatobiliary Cancers Clinical Practice Guidelines in Oncology. Available at http://www.nccn.org/professionals/physician_ gls/pdf/hepatobiliary.pdf.Version 2.2016.

20. Tabrizian P, Roayaie S and Schwartz ME: Current management of hepatocellular carcinoma. World J Gastroenterol 20: 10223-10237, 2014.

21. Maida M, Orlando E, Cammà C and Cabibbo G: Staging systems of hepatocellular carcinoma: A review of literature. World J Gastroenterol 20: 4141-4150, 2014.

22. Huang YH, Chen $\mathrm{CH}$, Chang TT, Chen SC, Wang SY, Lee HS, Lin PW, Huang GT, Sheu JC, Tsai HM, et al: Evaluation of predictive value of CLIP, Okuda, TNM and JIS staging systems for hepatocellular carcinoma patients undergoing surgery. J Gastroenterol Hepatol 20: 765-771, 2005. 
23. Huitzil-Melendez FD, Capanu M, O'Reilly EM, Duffy A, Gansukh B, Saltz LL and Abou-Alfa GK: Advanced hepatocellular carcinoma: Which staging systems best predict prognosis? J Clin Oncol 28: 2889-2895, 2010.

24. Kudo M, Chung H, Haji S, Osaki Y, Oka H, Seki T, Kasugai H, Sasaki Y and Matsunaga T: Validation of a new prognostic staging system for hepatocellular carcinoma: The JIS score compared with the CLIP score. Hepatology 40: 1396-1405, 2004.

25. Nishikawa H, Kita R, Kimura T, Endo M, Ohara Y, Sakamoto A Saito S, Nishijima N, Nasu A, Komekado H and Osaki Y: Proposal of the performance status combined Japan Integrated Staging system in hepatocellular carcinoma complicated with cirrhosis. Int J Oncol 46: 2371-2379, 2015.

26. Yen $\mathrm{YH}$, Changchien CS, Wang JH, Kee KM, Hung $\mathrm{CH}$, Hu TH, Lee CM, Lin CY, Wang CC, Chen TY, et al: A modified TNM-based Japan Integrated Score combined with AFP level may serve as a better staging system for early-stage predominant hepatocellular carcinoma patients. Dig Liver Dis 41: 431-441, 2009

27. Qin LX and Tang ZY: The prognostic significance of clinical and pathological features in hepatocellular carcinoma. World J Gastroenterol 8: 193-199, 2002.

28. Toyoda H, Kumada T, Osaki Y, Oka H, Urano F, Kudo M and Matsunaga T: Staging hepatocellular carcinoma by a novel scoring system (BALAD score) based on serum markers. Clin Gastroenterol Hepatol 4: 1528-1536, 2006.

29. Ji Y, Li B, Zhu Z, Guo X, He W, Fan Z and Zhang W: Overexpression of WAVE3 promotes tumor invasiveness and confers an unfavorable prognosis in human hepatocellular carcinoma. Biomed Pharmacother 69: 409-415, 2015.

30. Hashimoto R, Kanda M, Takami H, Shimizu D, Oya H, Hibino S, Okamura Y, Yamada S, Fujii T, Nakayama G, et al: Aberrant expression of melanoma-associated antigen-D2 serves as a prognostic indicator of hepatocellular carcinoma outcome following curative hepatectomy. Oncol Lett 9: 1201-1206, 2015.
31. Yuan SG, Liao WJ, Yang JJ, Huang GJ and Huang ZQ: DEP domain containing 1 is a novel diagnostic marker and prognostic predictor for hepatocellular carcinoma. Asian Pac J Cancer Prev 15: 10917-10922, 2014

32. Zhen L, Shijie N and Shuijun Z: Tumor PHD2 expression is correlated with clinical features and prognosis of patients with HCC receiving liver resection. Medicine (Baltimore) 93: e179, 2014.

33. Cai L and Cai X: Up-regulation of miR-9 expression predicate advanced clinicopathological features and poor prognosis in patients with hepatocellular carcinoma. Diagn Pathol 9: 1000, 2014.

34. Meng FL, Wang W and Jia WD: Diagnostic and prognostic significance of serum miR-24-3p in HBV-related hepatocellular carcinoma. Med Oncol 31: 177, 2014.

35. Heo MJ, Kim YM, Koo JH, Yang YM, An J, Lee SK, Lee SJ, Kim KM, Park JW and Kim SG: microRNA-148a dysregulation discriminates poor prognosis of hepatocellular carcinoma in association with USP4 overexpression. Oncotarget 5: 2792-2806, 2014.

36. Govaere O, Komuta M, Berkers J, Spee B, Janssen C, de Luca F, Katoonizadeh A, Wouters J, van Kempen LC, Durnez A, et al: Keratin 19: A key role player in the invasion of human hepatocellular carcinomas. Gut 63: 674-685, 2014.

37. Kaseb AO, Hassan MM, Lin E, Xiao L, Kumar V, Pathak P, Lozano R, Rashid A, Abbruzzese JL and Morris JS: V-CLIP: Integrating plasma vascular endothelial growth factor into a new scoring system to stratify patients with advanced hepatocellular carcinoma for clinical trials. Cancer 117: 2478-2488, 2011.

38. Nault JC, De Reyniès A, Villanueva A, Calderaro J, Rebouissou S, Couchy G, Decaens T, Franco D, Imbeaud S, Rousseau F, et al: A hepatocellular carcinoma 5-gene score associated with survival of patients after liver resection. Gastroenterology 145: 176-187, 2013. 